\title{
Gauge Fixing Identity in the Background Field Method of QCD in Pure Gauge
}

\author{
Gouranga C Nayak ${ }^{1, *}$ \\ ${ }^{1}$ Department of Physics, University of Arizona, Tucson, AZ 85721, USA
}

\begin{abstract}
In this paper we derive a gauge fixing identity by varying the covariant gauge fixing term in $Z[A, J, \eta, \bar{\eta}]$ in the background field method of QCD in pure gauge. Using this gauge fixing identity we establish a relation between $Z[J, \eta, \bar{\eta}]$ in QCD and $Z[A, J, \eta, \bar{\eta}]$ in background field method of QCD in pure gauge. We show the validity of this gauge fixing identity in general non-covariant and general Coulomb gauge fixings respectively. This gauge fixing identity is used to prove factorization theorem in QCD at high energy colliders and in non-equilibrium QCD at high energy heavy-ion colliders.

PACS numbers: PACS: 12.38.Lg,12.38.-t,12.38.Mh,11.15.Kc
\end{abstract}

*Electronic address: nayak@physics.arizona.edu 


\section{INTRODUCTION}

Background field method of QCD was originally formulated by 't Hooft [1] and later extended by Abbott [2]. This is an elegant formalism which can be useful to construct gauge invariant (off-shell) green's functions in QCD. For example, certain properties of structure functions and/or fragmentation functions at high energy colliders and at high energy heavy-ion colliders may be studied by using these gauge invariant green's functions. This formalism is also useful to study quark and gluon production from classical chromo field [3] via Schwinger mechanism [4], to compute $\beta$ function in QCD [5] and to study evolution of QCD coupling constant in the presence of chromofield [6].

Unlike QCD, since the lagrangian density in the generating functional in the background field method of QCD is gauge invariant (even after quantizing the theory), this formalism may be useful to study properties of certain gauge invariant non-perturbative physical quantities in QCD. From this point of view it is desirable to find situations where we can relate generating functional in the background field method of QCD to the generating functional in QCD (i.e. QCD without the background field). In the presence of external sources a relation between the generating functional in QCD and the generating functional in the background field method of QCD in pure gauge

$$
T^{a} A_{\mu}^{a}(x)=\frac{1}{i g}\left(\partial_{\mu} U\right) U^{-1}, \quad U=e^{i g T^{a} \beta^{a}(x)}
$$

may be useful in many physical situations.

However, unlike QED [7], finding an exact relation between the generating functional $Z[J, \eta, \bar{\eta}]$ in QCD and the generating functional $Z[A, J, \eta, \bar{\eta}]$ in the background field method of QCD in pure gauge is not easy. The main difficulty is due to the gauge fixing terms which are different in both the cases. While the Lorentz (covariant) gauge fixing term in QCD is independent of the background field $A_{\mu}^{a}$, the background field gauge fixing term in the background field method of QCD depends on $A_{\mu}^{a}[1,2]$. Hence it might be useful to obtain a gauge fixing identity by varying the gauge fixing term in $Z[A, J, \eta, \bar{\eta}]$ in the background field method of QCD in order to find a relation between $Z[J, \eta, \bar{\eta}]$ in $\mathrm{QCD}$ and $Z[A, J, \eta, \bar{\eta}]$ in background field method of QCD in pure gauge. In QCD without background field, a general gauge fixing WT identity was obtained in [8] .

In this paper we will derive a gauge fixing identity by varying the covariant gauge fixing term in $Z[A, J, \eta, \bar{\eta}]$ in the background field method of QCD in pure gauge. We will make a 
detailed analysis of this identity by using general non-covariant and general Coulomb gauge fixing terms. We will show that the gauge fixing identity holds for covariant, general noncovariant and general Coulomb gauge fixings respectively. Using this gauge fixing identity we will establish a relation between $Z[J, \eta, \bar{\eta}]$ in QCD and $Z[A, J, \eta, \bar{\eta}]$ in background field method of QCD in pure gauge.

We have used this gauge fixing identity in [9] to prove factorization of soft and collinear divergences at high energy colliders. We have also used this identity in [10] to prove factorization of fragmentation function in non-equilibrium QCD which can be experimentally applicable at high energy heavy-ion colliders at RHIC and LHC [11].

The paper is organized as follows. We derive a gauge fixing identity by varying the covariant gauge fixing term in $Z[A, J, \eta, \bar{\eta}]$ in background field method of QCD in pure gauge in section II. In section III and IV we perform our calculation by using general noncovariant and general Coulomb gauge fixings respectively. We establish a relation between $Z[J, \eta, \bar{\eta}]$ in QCD and $Z[A, J, \eta, \bar{\eta}]$ in background field method of QCD in pure gauge by using this gauge fixing identity in section V. Section VI contains conclusions.

\section{DERIVATION OF GAUGE FIXING IDENTITY BY USING COVARIANT GAUGE FIXING}

In the background field method of QCD the generating functional is given by [1, 2]

$$
\begin{aligned}
& Z^{G}[A, J, \eta, \bar{\eta}]=\int[d Q][d \bar{\psi}][d \psi] \operatorname{det}\left(\frac{\delta G^{a}(Q)}{\delta \omega^{b}}\right) \\
& e^{i \int d^{4} x\left[-\frac{1}{4} F^{a 2}{ }_{\mu \nu}[A+Q]-\frac{1}{2 \alpha}\left(G^{a}(Q)\right)^{2}+\bar{\psi} \not D[A+Q] \psi+J \cdot Q+\bar{\eta} \psi+\eta \bar{\psi}\right]}
\end{aligned}
$$

where the covariant gauge fixing term is

$$
G^{a}(Q)=\partial_{\mu} Q^{\mu a}+g f^{a b c} A_{\mu}^{b} Q^{\mu c}=D_{\mu}[A] Q^{\mu a}
$$

which depends on the background field $A_{\mu}^{a}$. Under the infinitesimal gauge transformation

$$
\beta<<1,
$$

the gluon field $Q_{\mu}^{a}$ and the background field $A_{\mu}^{a}$ transform as follows [2]

$$
\begin{aligned}
& \delta Q_{\mu}^{a}=-g f^{a b c} \beta^{b}\left(A_{\mu}^{c}+Q_{\mu}^{c}\right)+\partial_{\mu} \beta^{a} \\
& \delta A_{\mu}^{a}=0 .
\end{aligned}
$$


By changing $Q \rightarrow Q-A$ in eq. (2) we find

$$
\begin{aligned}
& Z^{G_{f}}[A, J, \eta, \bar{\eta}]=e^{-i \int d^{4} x J \cdot A} \int[d Q][d \bar{\psi}][d \psi] \operatorname{det}\left(\frac{\delta G_{f}^{a}(Q)}{\delta \beta^{b}}\right) \\
& e^{i \int d^{4} x\left[-\frac{1}{4} F_{\mu \nu}^{a 2}[Q]-\frac{1}{2 \alpha}\left(G_{f}^{a}(Q)\right)^{2}+\bar{\psi} \not[Q] \psi+J \cdot Q+\eta \bar{\psi}+\bar{\eta} \psi\right]}
\end{aligned}
$$

where the gauge fixing term from eq. (3) becomes

$$
G_{f}^{a}(Q)=\partial_{\mu} Q^{\mu a}+g f^{a b c} A_{\mu}^{b} Q^{\mu c}-\partial_{\mu} A^{\mu a}=D_{\mu}[A] Q^{\mu a}-\partial_{\mu} A^{\mu a},
$$

and eq. (5) becomes

$$
\delta Q_{\mu}^{a}=-g f^{a b c} \beta^{b} Q_{\mu}^{c}+\partial_{\mu} \beta^{a}
$$

Note that eq. (8) is precisely the gauge transformation in QCD without the background field. Hence we can anticipate a relation between QCD and the background field method of QCD when we relate $\beta^{a}(x)$ to the the background field $A_{\mu}^{a}(x)$. For a pure gauge, $\beta^{a}(x)$ is related to the background field $A_{\mu}^{a}(x)$ in eq. (1).

In QCD (without the background field) the covariant gauge fixing term is

$$
G_{n}^{a}(Q)=\partial_{\mu} Q^{\mu a}
$$

We write this as

$$
G_{n}^{a}(Q)=G_{f}^{a}(Q)+\Delta G_{f}^{a}(Q), \quad \quad \Delta G_{f}^{a}(Q)=-g f^{a b c} A_{\mu}^{b} Q^{\mu c}+\partial_{\mu} A^{\mu a}
$$

Using this in eq. (6) we find

$$
\begin{aligned}
& Z^{G_{f}+\Delta G_{f}}[A, J, \eta, \bar{\eta}]=e^{-i \int d^{4} x J \cdot A} \int[d Q][d \bar{\psi}][d \psi] \operatorname{det}\left(\frac{\delta\left(G_{f}^{a}(Q)+\Delta G_{f}^{a}(Q)\right)}{\delta \beta^{b}}\right) \\
& e^{i \int d^{4} x\left[-\frac{1}{4} F_{\mu \nu}^{a 2}[Q]-\frac{1}{2 \alpha}\left(G_{f}^{a}(Q)+\Delta G_{f}^{a}(Q)\right)^{2}+\bar{\psi} \not D[Q] \psi+J \cdot Q+\bar{\eta} \psi+\eta \bar{\psi}\right]} .
\end{aligned}
$$

The ghost determinant becomes

$$
\begin{aligned}
& \operatorname{det}\left(\frac{\delta\left(G_{f}^{a}(Q)+\Delta G_{f}^{a}(Q)\right)}{\delta \beta^{b}}\right)=\operatorname{det}\left(\frac{\delta G_{f}^{a}(Q)}{\delta \beta^{b}}\right)\left[1+\operatorname{tr}\left[\left(\frac{\delta \Delta G_{f}^{c}(Q)}{\delta \beta^{d}}\right)\left(\frac{\delta \beta^{d}}{\delta G_{f}^{e}(Q)}\right)\right]+\ldots\right] \\
& =\operatorname{det}\left(\frac{\delta G_{f}^{a}(Q)}{\delta \beta^{b}}\right)\left[1+\left(\frac{\delta \Delta G_{f}^{c}(Q)}{\delta \beta^{d}} \frac{\delta \beta^{d}}{\delta G_{f}^{c}(Q)}\right)+\ldots\right] .
\end{aligned}
$$

Using eqs. (12) and (마) in (111) we find

$$
\begin{aligned}
& Z^{G_{f}+\Delta G_{f}}[A, J, \eta, \bar{\eta}]=Z^{G_{f}}[A, J, \eta, \bar{\eta}] \\
& +e^{-i \int d^{4} x J \cdot A} \int[d Q][d \bar{\psi}][d \psi] \operatorname{det}\left(\frac{\delta G_{f}^{a}(Q)}{\delta \beta^{b}}\right) e^{i \int d^{4} x\left[-\frac{1}{4} F_{\mu \nu}^{a 2}[Q]-\frac{1}{2 \alpha}\left(G_{f}^{a}(Q)\right)^{2}+\bar{\psi} \not D[Q] \psi+J \cdot Q+\bar{\eta} \psi+\eta \bar{\psi}\right]} \\
& {\left[i \int d^{4} x\left[-\frac{1}{2 \alpha}\left(\Delta G_{f}^{c}(Q)\right)^{2}-\frac{1}{\alpha} G_{f}^{c}(Q) \Delta G_{f}^{c}(Q)\right]+\left(\frac{\delta \Delta G_{f}^{c}(Q)}{\delta \beta^{d}} \frac{\delta \beta^{d}}{\delta G_{f}^{c}(Q)}\right)+\ldots\right] .}
\end{aligned}
$$


For infinitesimal gauge transformation we find from eq. (8)

$$
Q_{\mu}^{\prime a}=Q_{\mu}^{a}-g f^{a b c} \beta^{b} Q_{\mu}^{c}+\partial_{\mu} \beta^{a}=Q_{\mu}^{a}+\delta Q_{\mu}^{a}
$$

Changing the variables of integration from unprimed to primed variables in eq. (6) we find

$$
\begin{aligned}
& Z^{G_{f}}[A, J, \eta, \bar{\eta}]=e^{-i \int d^{4} x J \cdot A} \int\left[d Q^{\prime}\right]\left[d \bar{\psi}^{\prime}\right]\left[d \psi^{\prime}\right] \\
& \operatorname{det}\left(\frac{\delta G_{f}^{a}\left(Q^{\prime}\right)}{\delta \beta^{b}}\right) e^{i \int d^{4} x\left[-\frac{1}{4} F^{a 2}{ }_{\mu \nu}\left[Q^{\prime}\right]-\frac{1}{2 \alpha}\left(G_{f}^{a}\left(Q^{\prime}\right)\right)^{2}+\bar{\psi}^{\prime} \not D\left[Q^{\prime}\right] \psi^{\prime}+J \cdot Q^{\prime}+\bar{\eta} \psi^{\prime}+\eta \bar{\psi}^{\prime}\right]} .
\end{aligned}
$$

This is because a change of variables from unprimed to primed variables does not change the value of the integration. Under the infinitesimal gauge transformation, using eq. (14), we find [12]

$$
\begin{aligned}
& {\left[d Q^{\prime}\right]=[d Q] \operatorname{det}\left[\frac{\partial Q^{\prime a}}{\partial Q^{b}}\right]=[d Q] \operatorname{det}\left[\delta^{a b}-g f^{a b c} \beta^{c}\right]} \\
& =[d Q]\left[1-\operatorname{tr} g f^{a b c} \beta^{c}+\mathcal{O}\left(\beta^{2}\right)+\ldots\right]=[d Q]\left[1+\mathcal{O}\left(\beta^{2}\right)+\ldots\right]=[d Q],
\end{aligned}
$$

where we have made use of eq. (4). Similarly under this infinitesimal gauge transformation the fermion fields transform accordingly i.e.

$$
\psi^{\prime}=\psi+\delta \psi, \quad \bar{\psi}^{\prime}=\bar{\psi}+\delta \bar{\psi}
$$

Using eqs. (14) and (17) we find

$$
\left[d \bar{\psi}^{\prime}\right]\left[d \psi^{\prime}\right]=[d \bar{\psi}][d \psi], \quad F_{\mu \nu}^{a 2}\left[Q^{\prime}\right]=F_{\mu \nu}^{a 2}[Q], \quad \bar{\psi}^{\prime} D^{\prime}\left[Q^{\prime}\right] \psi^{\prime}=\bar{\psi} D[Q] \psi
$$

which are gauge invariant.

Using eqs. (16) and (18) in eq. (15) we find

$$
\begin{aligned}
& Z^{G_{f}}[A, J, \eta, \bar{\eta}]=e^{-i \int d^{4} x J \cdot A} \int[d Q][d \bar{\psi}][d \psi] \\
& \operatorname{det}\left(\frac{\delta G_{f}^{a}\left(Q^{\prime}\right)}{\delta \beta^{b}}\right) e^{i \int d^{4} x\left[-\frac{1}{4} F_{\mu \nu}^{a 2}[Q]-\frac{1}{2 \alpha}\left(G_{f}^{a}\left(Q^{\prime}\right)\right)^{2}+\bar{\psi} \not[Q] \psi+J \cdot Q^{\prime}+\bar{\eta} \psi^{\prime}+\eta \bar{\psi}^{\prime}\right]}
\end{aligned}
$$

Let us consider the background field to be a pure gauge as given by eq. (1). For infinitesimal gauge transformation parameter $\beta$, see eq. (4), we find from eq. (1)

$$
A_{\mu}^{a}(x)=\partial_{\mu} \beta^{a}(x)
$$

From eq. (7) we find

$$
G_{f}^{a}\left(Q^{\prime}\right)=\partial_{\mu} Q^{\prime \mu a}+g f^{a b c} A_{\mu}^{b} Q^{\prime \mu c}-\partial_{\mu} A^{\mu a}
$$


which gives (by using eqs. (14) and (201)

$$
\begin{aligned}
& G_{f}^{a}\left(Q^{\prime}\right)=\partial_{\mu}\left[Q^{\mu a}-g f^{a b c} \beta^{b} Q^{\mu c}+\partial^{\mu} \beta^{a}\right]+g f^{a b c} A_{\mu}^{b}\left[Q^{\mu^{c}}-g f^{c d e} \beta^{d} Q^{\mu e}+\partial^{\mu} \beta^{c}\right]-\partial_{\mu} A^{\mu a} \\
& =\partial_{\mu} Q^{\mu a}-g f^{a b c}\left(\partial_{\mu} \beta^{b}\right) Q^{\mu c}-g f^{a b c} \beta^{b}\left(\partial_{\mu} Q^{\mu c}\right)+\partial_{\mu} \partial^{\mu} \beta^{a}+g f^{a b c} A_{\mu}^{b} Q^{\mu c}-g f^{a b c} A_{\mu}^{b} g f^{c d e} \beta^{d} Q^{\mu e} \\
& +g f^{a b c} A_{\mu}^{b} \partial^{\mu} \beta^{c}-\partial_{\mu} A^{\mu a} \\
& =\partial_{\mu} Q^{\mu a}-g f^{a b c} A_{\mu}^{b} Q^{\mu c}-g f^{a b c} \beta^{b}\left(\partial_{\mu} Q^{\mu c}\right)+\partial_{\mu} A^{\mu a}+g f^{a b c} A_{\mu}^{b} Q^{\mu c}-g f^{a b c} A_{\mu}^{b} g f^{c d e} \beta^{d} Q^{\mu e} \\
& +g f^{a b c} A_{\mu}^{b} A^{\mu c}-\partial_{\mu} A^{\mu a} \\
& =\partial_{\mu} Q^{\mu a}-g f^{a b c} \beta^{b}\left(\partial_{\mu} Q^{\mu c}\right)-g f^{a b c} A_{\mu}^{b} g f^{c d e} \beta^{d} Q^{\mu e} \\
& =\partial_{\mu} Q^{\mu a}-g f^{a b c} \beta^{b}\left(\partial_{\mu} Q^{\mu c}\right)+\mathcal{O}\left(\beta^{2}\right) .
\end{aligned}
$$

Note that $A$ is proportional to $\beta$, see eq. (20). Hence under an infinitesimal gauge transformation (using eq. (14)) we find from eq. (22)

$$
G_{f}^{a}\left(Q^{\prime}\right)=\partial_{\mu} Q^{\mu a}-g f^{a b c} \beta^{b}\left(\partial_{\mu} Q^{\mu c}\right)
$$

where we have kept terms up to order $\mathcal{O}(\beta)$ and have neglected terms of order $\mathcal{O}\left(\beta^{2}\right)$, see eq. (44). Eq. (23) gives

$$
\left(G_{f}^{a}\left(Q^{\prime}\right)\right)^{2}=\left(\partial_{\mu} Q^{\mu a}\right)^{2}-2 g f^{a b c}\left(\partial_{\nu} Q^{\nu a}\right) \beta^{b}\left(\partial_{\mu} Q^{\mu c}\right)=\left(\partial_{\mu} Q^{\mu a}\right)^{2}
$$

where we have kept terms up to order $\mathcal{O}(\beta)$ and have neglected terms of order $\mathcal{O}\left(\beta^{2}\right)$ using eq. (41). Hence we find

$$
\left(G_{f}^{a}\left(Q^{\prime}\right)\right)^{2}=\left(\partial_{\mu} Q^{\mu a}\right)^{2}=\left(G_{n}^{a}(Q)\right)^{2}=\left(G_{f}^{a}(Q)+\Delta G_{f}^{a}(Q)\right)^{2}
$$

where we have used eqs. (9) and (10). Using eq. (23) we find

$$
\operatorname{det} \frac{\delta G_{f}^{a}\left(Q^{\prime}\right)}{\delta \beta^{b}}=\operatorname{det} \frac{\delta\left[\partial_{\mu} Q^{\mu a}-g f^{a b c} \beta^{b}\left(\partial_{\mu} Q^{\mu c}\right)\right]}{\delta \beta^{b}}=\operatorname{det}\left[\frac{\delta \partial_{\mu} Q^{\mu a}}{\delta \beta^{b}}-\frac{\delta\left[g f^{a b^{\prime} c^{\prime}} \beta^{b^{\prime}}\left(\partial_{\mu} Q^{\mu c^{\prime}}\right)\right]}{\delta \beta^{b}}\right] .
$$

Since from eq. (8)

$$
\delta Q_{\mu}^{a} \propto \beta^{a}
$$

we find

$$
\delta\left[g f^{a b c} \beta^{b}\left(\partial_{\mu} Q^{\mu c}\right)\right] \propto\left(\beta^{a}\right)^{2} .
$$


Keeping terms up to order $\mathcal{O}(\beta)$ and neglecting terms of order $\mathcal{O}\left(\beta^{2}\right)$, see eq. (4), we find from eq. (26)

$$
\begin{aligned}
& \operatorname{det} \frac{\delta G_{f}^{a}\left(Q^{\prime}\right)}{\delta \beta^{b}}=\operatorname{det} \frac{\delta \partial_{\mu} Q^{\mu a}}{\delta \beta^{b}}=\operatorname{det} \frac{\delta\left[G_{f}^{a}(Q)+\Delta G_{f}^{a}(Q)\right]}{\delta \beta^{b}} \\
& =\operatorname{det} \frac{\delta G_{f}^{a}(Q)}{\delta \beta^{b}}\left[1+\frac{\delta \Delta G_{f}^{c}(Q)}{\delta \beta^{d}} \frac{\delta \beta^{d}}{\delta G_{f}^{c}(Q)}+\ldots\right]
\end{aligned}
$$

where we have used eqs. (9) and (10). Using eqs. (25), (29), (14) and (17) in eq. (19) we find

$$
\begin{aligned}
& Z^{G_{f}}[A, J, \eta, \bar{\eta}]=e^{-i \int d^{4} x J \cdot A} \int[d Q][d \bar{\psi}][d \psi] \operatorname{det}\left(\frac{\delta G_{f}^{a}(Q)}{\delta \beta^{b}}\right) \\
& e^{i \int d^{4} x\left[-\frac{1}{4} F_{\mu \nu}^{a 2}[Q]-\frac{1}{2 \alpha}\left(G_{f}^{a}(Q)\right)^{2}+\bar{\psi} \not D[Q] \psi+J \cdot Q+\bar{\eta} \psi+\eta \bar{\psi}\right]}\left[1+i \int d^{4} x\left[-\frac{1}{\alpha} G_{f}^{c}(Q) \Delta G_{f}^{c}(Q)-\frac{1}{2 \alpha}\left(\Delta G_{f}^{c}(Q)\right)^{2}\right.\right. \\
& \left.+J \cdot \delta Q+\bar{\eta} \delta \psi+\eta \delta \bar{\psi}]+\frac{\delta \Delta G_{f}^{c}(Q)}{\delta \beta^{d}} \frac{\delta \beta^{d}}{\delta G_{f}^{c}(Q)}+\ldots\right] .
\end{aligned}
$$

Using eq. (6) in (30) we find

$$
\begin{aligned}
& Z^{G_{f}}[A, J, \eta, \bar{\eta}]=Z^{G_{f}}[A, J, \eta, \bar{\eta}] \\
& +e^{-i \int d^{4} x J \cdot A} \int[d Q][d \bar{\psi}][d \psi] \operatorname{det}\left(\frac{\delta G_{f}^{a}(Q)}{\delta \beta^{b}}\right) e^{i \int d^{4} x\left[-\frac{1}{4} F_{\mu \nu}^{a 2}[Q]-\frac{1}{2 \alpha}\left(G_{f}^{a}(Q)\right)^{2}+\bar{\psi} \not D[Q] \psi+J \cdot Q+\bar{\eta} \psi+\eta \bar{\psi}\right]} \\
& {\left[i \int d^{4} x\left[-\frac{1}{\alpha} G_{f}^{a}(Q) \Delta G_{f}^{a}(Q)-\frac{1}{2 \alpha}\left(\Delta G_{f}^{a}(Q)\right)^{2}+J \cdot \delta Q+\bar{\eta} \delta \psi+\eta \delta \bar{\psi}\right]+\frac{\delta \Delta G_{f}^{c}(Q)}{\delta \beta^{d}} \frac{\delta \beta^{d}}{\delta G_{f}^{c}(Q)}+\ldots\right] .}
\end{aligned}
$$

From the above equation we obtain our required identity

$$
\begin{aligned}
& e^{-i \int d^{4} x J \cdot A} \int[d Q][d \bar{\psi}][d \psi] \operatorname{det}\left(\frac{\delta G_{f}^{a}(Q)}{\delta \beta^{b}}\right) e^{i \int d^{4} x\left[-\frac{1}{4} F_{\mu \nu}^{a 2}[Q]-\frac{1}{2 \alpha}\left(G_{f}^{a}(Q)\right)^{2}+\bar{\psi} \not D[Q] \psi+J \cdot Q+\bar{\eta} \psi+\eta \bar{\psi}\right]} \\
& {\left[i \int d^{4} x\left[-\frac{1}{\alpha} G_{f}^{c}(Q) \Delta G_{f}^{c}(Q)-\frac{1}{2 \alpha}\left(\Delta G_{f}^{c}(Q)\right)^{2}+J \cdot \delta Q+\bar{\eta} \delta \psi+\eta \delta \bar{\psi}\right]\right.} \\
& \left.+\frac{\delta \Delta G_{f}^{c}(Q)}{\delta \beta^{d}} \frac{\delta \beta^{d}}{\delta G_{f}^{c}(Q)}+\ldots\right]=0
\end{aligned}
$$

Eq. (32) is the gauge fixing identity by varying the gauge fixing term in $Z[A, J, \eta, \bar{\eta}]$ in the background field method of QCD in pure gauge. 


\section{DERIVATION OF GAUGE FIXING IDENTITY BY USING GENERAL NON-COVARIANT GAUGE FIXING}

The generating functional in the background field method of QCD with general noncovariant gauge fixing is given by

$$
\begin{aligned}
& Z^{G}[A, J, \eta, \bar{\eta}]=\int[d Q][d \bar{\psi}][d \psi] \operatorname{det}\left(\frac{\delta G^{a}(Q)}{\delta \omega^{b}}\right) \\
& e^{i \int d^{4} x\left[-\frac{1}{4} F_{\mu \nu}^{a 2}[A+Q]-\frac{1}{2 \alpha}\left(G^{a}(Q)\right)^{2}+\bar{\psi} \not D[A+Q] \psi+J \cdot Q+\bar{\eta} \psi+\eta \bar{\psi}\right]}
\end{aligned}
$$

where

$$
G^{a}(Q)=\frac{\eta^{\mu} \eta^{\nu}}{\eta^{2}}\left(\partial_{\mu} Q_{\nu}^{a}+g f^{a b c} A_{\mu}^{b} Q_{\nu}^{c}\right)=\frac{\eta^{\mu} \eta^{\nu}}{\eta^{2}} D_{\mu}[A] Q_{\nu}^{a}
$$

is the gauge fixing term in general non-covariant gauges [13, 14] with $\eta^{\mu}$ being an arbitrary but constant four vector. By changing $Q_{\mu}^{a} \rightarrow Q_{\mu}^{a}-A_{\mu}^{a}$ in eq. (33) we find

$$
\begin{aligned}
& Z^{G_{f}}[A, J, \eta, \bar{\eta}]=e^{-i \int d^{4} x J \cdot A} \int[d Q][d \bar{\psi}][d \psi] \operatorname{det}\left(\frac{\delta G_{f}^{a}(Q)}{\delta \beta^{b}}\right) \\
& e^{i \int d^{4} x\left[-\frac{1}{4} F^{a 2}{ }_{\mu \nu}[Q]-\frac{1}{2 \alpha}\left(G_{f}^{a}(Q)\right)^{2}+\bar{\psi} \not D[Q] \psi+J \cdot Q+\eta \bar{\psi}+\bar{\eta} \psi\right]}
\end{aligned}
$$

where

$$
\begin{aligned}
& G_{f}^{a}(Q)=\frac{\eta^{\mu} \eta^{\nu}}{\eta^{2}}\left(\partial_{\mu} Q_{\nu}^{a}+g f^{a b c} A_{\mu}^{b} Q_{\nu}^{c}-\partial_{\mu} A_{\nu}^{a}\right)-\frac{1}{\eta^{2}} g f^{a b c}\left(\eta \cdot A^{b}\right)\left(\eta \cdot A^{c}\right) \\
& =\frac{\eta^{\mu} \eta^{\nu}}{\eta^{2}}\left(D_{\mu}[A] Q_{\nu}^{a}\right)-\frac{\eta^{\mu} \eta_{\nu}}{\eta^{2}} \partial_{\mu} A_{\nu}^{a} .
\end{aligned}
$$

In QCD (without the background field) the generating functional with general non-covariant gauge fixing is given by

$$
\begin{aligned}
& Z[J, \eta, \bar{\eta}]=\int[d Q][d \bar{\psi}][d \psi] \operatorname{det}\left(\frac{\delta G_{n}^{a}(Q)}{\delta \beta^{b}}\right) \\
& e^{i \int d^{4} x\left[-\frac{1}{4} F^{a 2}{ }_{\mu \nu}[Q]-\frac{1}{2 \alpha}\left(G_{n}^{a}(Q)\right)^{2}+\bar{\psi} \not D[Q] \psi+J \cdot Q+\eta \bar{\psi}+\bar{\eta} \psi\right]}
\end{aligned}
$$

where

$$
G_{n}^{a}(Q)=\frac{\eta^{\mu} \eta^{\nu}}{\eta^{2}} \partial_{\mu} Q_{\nu}^{a}
$$

is the gauge fixing term in general non-covariant gauges [13, 14]. We write this as

$$
G_{n}^{a}(Q)=G_{f}^{a}(Q)+\Delta G_{f}^{a}(Q), \quad \quad \Delta G_{f}^{a}(Q)=\frac{\eta^{\mu} \eta^{\nu}}{\eta^{2}}\left(-g f^{a b c} A_{\mu}^{b} Q_{\nu}^{c}+\partial_{\mu} A_{\nu}^{a}\right)
$$


Changing the variables of integration from unprimed to primed variables in eq. (35) we find

$$
\begin{aligned}
& Z^{G_{f}}[A, J, \eta, \bar{\eta}]=e^{-i \int d^{4} x J \cdot A} \int[d Q][d \bar{\psi}][d \psi] \\
& \operatorname{det}\left(\frac{\delta G_{f}^{a}\left(Q^{\prime}\right)}{\delta \beta^{b}}\right) e^{i \int d^{4} x\left[-\frac{1}{4} F_{\mu \nu}^{a 2}[Q]-\frac{1}{2 \alpha}\left(G_{f}^{a}\left(Q^{\prime}\right)\right)^{2}+\bar{\psi} \not D[Q] \psi+J \cdot Q^{\prime}+\bar{\eta} \psi^{\prime}+\eta \bar{\psi}^{\prime}\right]}
\end{aligned}
$$

which is similar to eq. (19) but with $G_{f}^{a}(Q)$ given by eq. (36).

By using eqs. (14) and (20) we find from eq. (36)

$$
\begin{aligned}
& G_{f}^{a}\left(Q^{\prime}\right)=\frac{\eta^{\mu} \eta_{\nu}}{\eta^{2}}\left(\partial_{\mu}\left[Q^{\nu a}-g f^{a b c} \beta^{b} Q^{\nu c}+\partial^{\nu} \beta^{a}\right]+g f^{a b c} A_{\mu}^{b}\left[Q^{\nu c}-g f^{c d e} \beta^{d} Q^{\nu e}+\partial^{\nu} \beta^{c}\right]-\partial_{\mu} A^{\nu a}\right) \\
& =\frac{\eta^{\mu} \eta_{\nu}}{\eta^{2}}\left(\partial_{\mu} Q^{\nu a}-g f^{a b c}\left(\partial_{\mu} \beta^{b}\right) Q^{\nu c}-g f^{a b c} \beta^{b}\left(\partial_{\mu} Q^{\nu c}\right)+\partial_{\mu} \partial^{\nu} \beta^{a}+g f^{a b c} A_{\mu}^{b} Q^{\nu c}-g f^{a b c} A_{\mu}^{b} g f^{c d e} \beta^{d} Q^{\nu e}\right. \\
& \left.+g f^{a b c} A_{\mu}^{b} \partial^{\nu} \beta^{c}-\partial_{\mu} A^{\nu a}\right) \\
& =\frac{\eta^{\mu} \eta_{\nu}}{\eta^{2}}\left(\partial_{\mu} Q^{\nu a}-g f^{a b c} A_{\mu}^{b} Q^{\nu c}-g f^{a b c} \beta^{b}\left(\partial_{\mu} Q^{\nu c}\right)+\partial_{\mu} A^{\nu a}+g f^{a b c} A_{\mu}^{b} Q^{\nu c}-g f^{a b c} A_{\mu}^{b} g f^{c d e} \beta^{d} Q^{\nu e}\right. \\
& \left.+g f^{a b c} A_{\mu}^{b} A^{\nu c}-\partial_{\mu} A^{\nu a}\right) \\
& =\frac{\eta^{\mu} \eta_{\nu}}{\eta^{2}}\left(\partial_{\mu} Q^{\nu a}-g f^{a b c} \beta^{b}\left(\partial_{\mu} Q^{\nu c}\right)-g f^{a b c} A_{\mu}^{b} g f^{c d e} \beta^{d} Q^{\nu e}\right)+\frac{1}{\eta^{2}} g f^{a b c}\left(\eta \cdot A^{b}\right)\left(\eta \cdot A^{c}\right) \\
& =\frac{\eta^{\mu} \eta_{\nu}}{\eta^{2}}\left(\partial_{\mu} Q^{\nu a}-g f^{a b c} \beta^{b}\left(\partial_{\mu} Q^{\nu c}\right)-g f^{a b c} A_{\mu}^{b} g f^{c d e} \beta^{d} Q^{\nu e}\right) \\
& =\frac{\eta^{\mu} \eta_{\nu}}{\eta^{2}}\left(\partial_{\mu} Q^{\nu a}-g f^{a b c} \beta^{b}\left(\partial_{\mu} Q^{\nu c}\right)\right)+\mathcal{O}\left(\beta^{2}\right) .
\end{aligned}
$$

Note that $A$ is proportional to $\beta$, see eq. (20). Hence under an infinitesimal gauge transformation (using eq. (14)) we find from eq. (41)

$$
G_{f}^{a}\left(Q^{\prime}\right)=\frac{\eta^{\mu} \eta_{\nu}}{\eta^{2}}\left(\partial_{\mu} Q^{\nu a}-g f^{a b c} \beta^{b}\left(\partial_{\mu} Q^{\nu c}\right)\right)
$$

where we have kept terms up to order $\mathcal{O}(\beta)$ and have neglected terms of order $\mathcal{O}\left(\beta^{2}\right)$, see eq. (44). Eq. (42) gives

$$
\left(G_{f}^{a}\left(Q^{\prime}\right)\right)^{2}=\left(\frac{\eta^{\mu} \eta_{\nu}}{\eta^{2}} \partial_{\mu} Q^{\nu a}\right)^{2}-2 g f^{a b c} \beta^{b} \frac{1}{\eta^{4}}\left(\eta \cdot \partial \eta \cdot Q^{c}\right)\left(\eta \cdot \partial \eta \cdot Q^{a}\right)+\mathcal{O}\left(\beta^{2}\right)
$$

Keeping terms up to order $\mathcal{O}(\beta)$ and neglecting terms of order $\mathcal{O}\left(\beta^{2}\right)$, see eq. (4), we find from eq. (43)

$$
\left(G_{f}^{a}\left(Q^{\prime}\right)\right)^{2}=\left(\frac{\eta^{\mu} \eta_{\nu}}{\eta^{2}} \partial_{\mu} Q^{\nu a}\right)^{2}=\left(G_{n}^{a}(Q)\right)^{2}=\left(G_{f}^{a}(Q)+\Delta G_{f}^{a}(Q)\right)^{2}
$$

where we have used eqs. (38) and (39). Using eq. (42) we find

$$
\operatorname{det} \frac{\delta G_{f}^{a}\left(Q^{\prime}\right)}{\delta \beta^{b}}=\operatorname{det} \frac{\frac{\eta^{\mu} \eta_{\nu}}{\eta^{2}}\left(\delta\left[\partial_{\mu} Q^{\nu a}-g f^{a b c} \beta^{b}\left(\partial_{\mu} Q^{\nu c}\right)\right]\right)}{\delta \beta^{b}}=\operatorname{det}\left[\frac{\eta^{\mu} \eta_{\nu}}{\eta^{2}}\left(\frac{\delta \partial_{\mu} Q^{\nu a}}{\delta \beta^{b}}-\frac{\delta\left[g f^{a b^{\prime} c^{\prime}} \beta^{b^{\prime}}\left(\partial_{\mu} Q^{\nu c^{\prime}}\right)\right]}{\delta \beta^{b}}\right)\right] .
$$


Keeping terms up to order $\mathcal{O}(\beta)$ and neglecting terms of order $\mathcal{O}\left(\beta^{2}\right)$, see eqs. (44) and (28), we find from eq. (45)

$$
\begin{aligned}
& \operatorname{det} \frac{\delta G_{f}^{a}\left(Q^{\prime}\right)}{\delta \beta^{b}}=\operatorname{det} \frac{\frac{\eta^{\mu} \eta_{\nu}}{\eta^{2}}\left(\delta \partial_{\mu} Q^{\nu a}\right)}{\delta \beta^{b}}=\operatorname{det} \frac{\delta\left[G_{f}^{a}(Q)+\Delta G_{f}^{a}(Q)\right]}{\delta \beta^{b}} \\
& =\operatorname{det} \frac{\delta G_{f}^{a}(Q)}{\delta \beta^{b}}\left[1+\frac{\delta \Delta G_{f}^{c}(Q)}{\delta \beta^{d}} \frac{\delta \beta^{d}}{\delta G_{f}^{c}(Q)}+\ldots\right]
\end{aligned}
$$

where we have used eqs. (38) and (39). Using eqs. (44), (46), (14) and (17) in eq. (40) we find

$$
\begin{aligned}
& Z^{G_{f}}[A, J, \eta, \bar{\eta}]=e^{-i \int d^{4} x J \cdot A} \int[d Q][d \bar{\psi}][d \psi] \operatorname{det}\left(\frac{\delta G_{f}^{a}(Q)}{\delta \beta^{b}}\right) \\
& e^{i \int d^{4} x\left[-\frac{1}{4} F^{a 2}{ }_{\mu \nu}[Q]-\frac{1}{2 \alpha}\left(G_{f}^{a}(Q)\right)^{2}+\bar{\psi} \not[Q] \psi+J \cdot Q+\bar{\eta} \psi+\eta \bar{\psi}\right]}\left[1+i \int d^{4} x\left[-\frac{1}{\alpha} G_{f}^{c}(Q) \Delta G_{f}^{c}(Q)-\frac{1}{2 \alpha}\left(\Delta G_{f}^{c}(Q)\right)^{2}\right.\right. \\
& \left.+J \cdot \delta Q+\bar{\eta} \delta \psi+\eta \delta \bar{\psi}]+\frac{\delta \Delta G_{f}^{c}(Q)}{\delta \beta^{d}} \frac{\delta \beta^{d}}{\delta G_{f}^{c}(Q)}+\ldots\right]
\end{aligned}
$$

where $G_{f}^{a}(Q)$ is given by eq. (36). Using eq. (33) we find from eq. (47)

$$
\begin{aligned}
& Z^{G_{f}}[A, J, \eta, \bar{\eta}]=Z^{G_{f}}[A, J, \eta, \bar{\eta}] \\
& +e^{-i \int d^{4} x J \cdot A} \int[d Q][d \bar{\psi}][d \psi] \operatorname{det}\left(\frac{\delta G_{f}^{a}(Q)}{\delta \beta^{b}}\right) e^{i \int d^{4} x\left[-\frac{1}{4} F_{\mu \nu}^{a 2}[Q]-\frac{1}{2 \alpha}\left(G_{f}^{a}(Q)\right)^{2}+\bar{\psi} \not[Q] \psi+J \cdot Q+\bar{\eta} \psi+\eta \bar{\psi}\right]} \\
& {\left[i \int d^{4} x\left[-\frac{1}{\alpha} G_{f}^{a}(Q) \Delta G_{f}^{a}(Q)-\frac{1}{2 \alpha}\left(\Delta G_{f}^{a}(Q)\right)^{2}+J \cdot \delta Q+\bar{\eta} \delta \psi+\eta \delta \bar{\psi}\right]+\frac{\delta \Delta G_{f}^{c}(Q)}{\delta \beta^{d}} \frac{\delta \beta^{d}}{\delta G_{f}^{c}(Q)}+\ldots\right] .}
\end{aligned}
$$

From the above equation we obtain our required gauge fixing identity

$$
\begin{aligned}
& e^{-i \int d^{4} x J \cdot A} \int[d Q][d \bar{\psi}][d \psi] \operatorname{det}\left(\frac{\delta G_{f}^{a}(Q)}{\delta \beta^{b}}\right) e^{i \int d^{4} x\left[-\frac{1}{4} F_{\mu \nu}^{a 2}[Q]-\frac{1}{2 \alpha}\left(G_{f}^{a}(Q)\right)^{2}+\bar{\psi} \not D[Q] \psi+J \cdot Q+\bar{\eta} \psi+\eta \bar{\psi}\right]} \\
& {\left[i \int d^{4} x\left[-\frac{1}{\alpha} G_{f}^{c}(Q) \Delta G_{f}^{c}(Q)-\frac{1}{2 \alpha}\left(\Delta G_{f}^{c}(Q)\right)^{2}+J \cdot \delta Q+\bar{\eta} \delta \psi+\eta \delta \bar{\psi}\right]\right.} \\
& \left.+\frac{\delta \Delta G_{f}^{c}(Q)}{\delta \beta^{d}} \frac{\delta \beta^{d}}{\delta G_{f}^{c}(Q)}+\ldots\right]=0
\end{aligned}
$$

by using general non-covariant gauge fixing.

Eq. (49) is exactly same as eq. (32) in covariant gauge fixing except that $G_{f}^{a}(Q)$ replaced by the general non-covariant gauge fixing term given by eq. (36). This completes the derivation of gauge fixing identity by using the general non-covariant gauge fixing. 


\section{DERIVATION OF GAUGE FIXING IDENTITY BY USING GENERAL COULOMB GAUGE FIXING}

The generating functional in the background field method of QCD with general Coulomb gauge fixing is given by

$$
\begin{aligned}
& Z^{G}[A, J, \eta, \bar{\eta}]=\int[d Q][d \bar{\psi}][d \psi] \operatorname{det}\left(\frac{\delta G^{a}(Q)}{\delta \omega^{b}}\right) \\
& e^{i \int d^{4} x\left[-\frac{1}{4} F_{\mu \nu}^{a}{ }_{\mu \nu}^{2}[A+Q]-\frac{1}{2 \alpha}\left(G^{a}(Q)\right)^{2}+\bar{\psi} \not D[A+Q] \psi+J \cdot Q+\bar{\eta} \psi+\eta \bar{\psi}\right]}
\end{aligned}
$$

where

$$
G^{a}(Q)=\left[g^{\mu \nu}-\frac{n^{\mu} n^{\nu}}{n^{2}}\right]\left(\partial_{\mu} Q_{\nu}^{a}+g f^{a b c} A_{\mu}^{b} Q_{\nu}^{c}\right)=\left[g^{\mu \nu}-\frac{\eta^{\mu} n^{\nu}}{n^{2}}\right] D_{\mu}[A] Q_{\nu}^{a}
$$

is the gauge fixing term in general Coulomb gauge [13] with

$$
n^{\mu}=(1,0,0,0)
$$

By changing $Q_{\mu}^{a} \rightarrow Q_{\mu}^{a}-A_{\mu}^{a}$ in eq. (50) we find

$$
\begin{aligned}
& Z^{G_{f}}[A, J, \eta, \bar{\eta}]=e^{-i \int d^{4} x J \cdot A} \int[d Q][d \bar{\psi}][d \psi] \operatorname{det}\left(\frac{\delta G_{f}^{a}(Q)}{\delta \beta^{b}}\right) \\
& e^{i \int d^{4} x\left[-\frac{1}{4} F_{\mu \nu}^{a 2}[Q]-\frac{1}{2 \alpha}\left(G_{f}^{a}(Q)\right)^{2}+\bar{\psi} \not D[Q] \psi+J \cdot Q+\eta \bar{\psi}+\bar{\eta} \psi\right]}
\end{aligned}
$$

where

$$
\begin{aligned}
& G_{f}^{a}(Q)=\left[g^{\mu \nu}-\frac{n^{\mu} n^{\nu}}{n^{2}}\right]\left(\partial_{\mu} Q_{\nu}^{a}+g f^{a b c} A_{\mu}^{b} Q_{\nu}^{c}-\partial_{\mu} A_{\nu}^{a}\right)-g f^{a b c} A_{\mu}^{b} A^{\mu c}+\frac{1}{n^{2}} g f^{a b c}\left(n \cdot A^{b}\right)\left(n \cdot A^{c}\right) \\
& =\left[g^{\mu \nu}-\frac{n^{\mu} n^{\nu}}{n^{2}}\right]\left(D_{\mu}[A] Q_{\nu}^{a}\right)-\left[g^{\mu \nu}-\frac{n^{\mu} n^{\nu}}{n^{2}}\right] \partial_{\mu} A_{\nu}^{a} .
\end{aligned}
$$

In QCD (without the background field) the generating functional with general Coulomb gauge fixing is given by

$$
Z[J, \eta, \bar{\eta}]=\int[d Q][d \bar{\psi}][d \psi] \operatorname{det}\left(\frac{\delta G_{n}^{a}(Q)}{\delta \beta^{b}}\right) e^{i \int d^{4} x\left[-\frac{1}{4} F_{\mu \nu}^{a 2}[Q]-\frac{1}{2 \alpha}\left(G_{n}^{a}(Q)\right)^{2}+\bar{\psi} \not D[Q] \psi+J \cdot Q+\eta \bar{\psi}+\bar{\eta} \psi\right]}
$$

where

$$
G_{n}^{a}(Q)=\left[g^{\mu \nu}-\frac{n^{\mu} n^{\nu}}{n^{2}}\right] \partial_{\mu} Q_{\nu}^{a}
$$

is the gauge fixing term in general Coulomb gauge [13]. 
Hence by replacing $\frac{\eta^{\mu} \eta^{\nu}}{\eta^{2}} \rightarrow\left[g^{\mu \nu}-\frac{n^{\mu} n^{\nu}}{n^{2}}\right]$ everywhere in the derivations in the previous section we find

$$
\begin{aligned}
& e^{-i \int d^{4} x J \cdot A} \int[d Q][d \bar{\psi}][d \psi] \operatorname{det}\left(\frac{\delta G_{f}^{a}(Q)}{\delta \beta^{b}}\right) e^{i \int d^{4} x\left[-\frac{1}{4} F_{\mu \nu}^{a 2}[Q]-\frac{1}{2 \alpha}\left(G_{f}^{a}(Q)\right)^{2}+\bar{\psi} \not \supset[Q] \psi+J \cdot Q+\bar{\eta} \psi+\eta \bar{\psi}\right]} \\
& {\left[i \int d^{4} x\left[-\frac{1}{\alpha} G_{f}^{c}(Q) \Delta G_{f}^{c}(Q)-\frac{1}{2 \alpha}\left(\Delta G_{f}^{c}(Q)\right)^{2}+J \cdot \delta Q+\bar{\eta} \delta \psi+\eta \delta \bar{\psi}\right]\right.} \\
& \left.+\frac{\delta \Delta G_{f}^{c}(Q)}{\delta \beta^{d}} \frac{\delta \beta^{d}}{\delta G_{f}^{c}(Q)}+\ldots\right]=0
\end{aligned}
$$

by using general Coulomb gauge fixing.

Eq. (57) is exactly same as eqs. (32) (or eq. (49)) with covariant gauge fixing (or general non-covariant gauge fixing) except that $G_{f}^{a}(Q)$ is replaced by general Coulomb gauge fixing term given by eq. (54) . This completes the derivation of gauge fixing identity by using the general Coulomb gauge fixing.

\section{RELATION BETWEEN $Z[J, \eta, \bar{\eta}]$ AND $Z[A, J, \eta, \bar{\eta}]$ IN PURE GAUGE}

Using eq. (9) and (10) in (6) we obtain

$$
\begin{aligned}
& Z^{G_{f}+\Delta G_{f}}[A, J, \eta, \bar{\eta}]=e^{-i \int d^{4} x J \cdot A} \int[d Q][d \bar{\psi}][d \psi] \operatorname{det}\left(\frac{\delta G_{n}^{a}(Q)}{\delta \beta^{b}}\right) \\
& e^{i \int d^{4} x\left[-\frac{1}{4} F_{\mu \nu}^{a 2}[Q]-\frac{1}{2 \alpha}\left(G_{n}^{a}(Q)\right)^{2}+\bar{\psi} \not D[Q] \psi+J \cdot Q+\bar{\eta} \psi+\eta \bar{\psi}\right]}=e^{-i \int d^{4} x J \cdot A} \times Z_{\mathrm{QCD}}[J, \eta, \bar{\eta}]
\end{aligned}
$$

where $Z_{\mathrm{QCD}}[J, \eta, \bar{\eta}]$ is the generating functional in QCD without the background field. Subtracting eq. (32) from eq. (13) we find

$$
\begin{aligned}
& Z^{G_{f}+\Delta G_{f}}[A, J, \eta, \bar{\eta}]=Z^{G_{f}}[A, J, \eta, \bar{\eta}]-e^{-i \int d^{4} x J \cdot A} \int[d Q][d \bar{\psi}][d \psi] \operatorname{det}\left(\frac{\delta G_{f}^{a}(Q)}{\delta \beta^{b}}\right) \\
& e^{i \int d^{4} x\left[-\frac{1}{4} F_{\mu \nu}^{a 2}[Q]-\frac{1}{2 \alpha}\left(G_{f}^{a}(Q)\right)^{2}+\bar{\psi} \not D[Q] \psi+J \cdot Q+\bar{\eta} \psi+\eta \bar{\psi}\right]}\left[i \int d^{4} x[J \cdot \delta Q+\bar{\eta} \delta \psi+\eta \delta \bar{\psi}+\ldots]\right] .
\end{aligned}
$$

From eq. (6) we denote

$$
Z_{\text {background } \mathrm{QCD}}[A, J, \eta, \bar{\eta}]=Z^{G_{f}}[A, J, \eta, \bar{\eta}]
$$

Hence from eqs. (6)

$$
\begin{aligned}
& Z_{\mathrm{QCD}}[J, \eta, \bar{\eta}]=e^{i \int d^{4} x J \cdot A} \times Z_{\text {background } \mathrm{QCD}}[A, J, \eta, \bar{\eta}]-\int[d Q][d \bar{\psi}][d \psi] \operatorname{det}\left(\frac{\delta G_{f}^{a}(Q)}{\delta \beta^{b}}\right) \\
& e^{i \int d^{4} x\left[-\frac{1}{4} F_{\mu \nu}^{a 2}[Q]-\frac{1}{2 \alpha}\left(G_{f}^{a}(Q)\right)^{2}+\bar{\psi} \not D[Q] \psi+J \cdot Q+\bar{\eta} \psi+\eta \bar{\psi}\right]}\left[i \int d^{4} x[J \cdot \delta Q+\bar{\eta} \delta \psi+\eta \delta \bar{\psi}+\ldots]\right]
\end{aligned}
$$


where the covariant gauge fixing term $G_{f}^{a}(Q)$ is given by eq. (77).

Similarly using eq. (49) with general non-covariant gauge fixing we arrive at eq. (61) with general non-covariant gauge fixing term $G_{f}^{a}(Q)$ given by eq. (36). Using eq. (57) with general Coulomb gauge fixing we arrive at eq. (61) with general Coulomb gauge fixing term $G_{f}^{a}(Q)$ given by eq. (54).

Hence we find that eq. (61) is the relation between generating functional in QCD and generating functional in the background field method of QCD in pure gauge with covariant, general non-covariant and general Coulomb gauge fixings respectively. We have used eq. (61) in [9] to prove factorization of soft and collinear divergences at high energy colliders. We have also used this equation in [10] is to prove factorization of fragmentation function in non-equilibrium QCD which can be experimentally applicable at RHIC and LHC [11].

\section{CONCLUSIONS}

Establishing a relation between $Z[A, J, \eta, \bar{\eta}]$ in the background field method of QCD in pure gauge and $Z[J, \eta, \bar{\eta}]$ in $\mathrm{QCD}$ can be useful in studying properties of certain nonperturbative physical quantities in QCD. However, unlike QED, establishing a relation between these two is not easy because the gauge fixing term in QCD is different from background field gauge fixing term in the background field method of QCD. For this purpose we have derived a gauge fixing identity by varying the covariant gauge fixing term in $Z[A, J, \eta, \bar{\eta}]$ in the background field method of QCD in pure gauge. We have made a detailed analysis of this gauge fixing identity by using general non-covariant and general Coulomb gauge fixing terms. We have found that the gauge fixing identity holds for covariant, general non-covariant and general Coulomb gauge fixings respectively.

Using this gauge fixing identity we have established a relation (given by eq. (61)) between $Z[J, \eta, \bar{\eta}]$ in QCD and $Z[A, J, \eta, \bar{\eta}]$ in background field method of QCD in pure gauge. We have used this gauge fixing identity in [9] to prove factorization of soft and collinear divergences at high energy colliders. We have also used this identity in [10] to prove factorization of fragmentation function in non-equilibrium QCD which can be experimentally applicable at RHIC and LHC [11]. 


\section{Acknowledgments}

I thank Peter van Nieuwenhuizen for useful discussions. This work was supported in part by Department of Energy under contracts DE-FG02-91ER40664, DE-FG02-04ER41319 and DE-FG02-04ER41298.

[1] G. 't Hooft, Nucl. Phys. B62 (1973) 444.

[2] L. F. Abbott, Nucl. Phys. B185 (1981) 189.

[3] G. C. Nayak and P. van Nieuwenhuizen, Phys. Rev. D71 (2005) 125001; F. Cooper and G. C. Nayak, Phys. Rev. D73 (2006) 065005; G. C. Nayak, Phys. Rev. D72 (2005) 125010.

[4] J. Schwinger, Phys. Rev. 82 (1951) 664; W. Heisenberg and H. Euler, Z. Physik 98, 714 (1936);

A. Casher, H. Neuberger and S. Nussinov, Phys. Rev. D 20, 179 (1979).

[5] M. E. Peskin and D. V. Schroeder, Introduction to Quantum Field Theory, Perseus Books Publishing, L.L.C.

[6] G. C. Nayak, Eur. Phys. J.C64:73,2009, arXiv:0812.5054 [hep-ph].

[7] R. Tucci, Phys. Rev. D32 (1985) 945.

[8] B. Lee and J. Zinn-Justin, Phys. Rev. D7 (1973) 1049.

[9] G. C. Nayak, Annals Phys. 325 (2010) 514, arXiv:0905.3923 [hep-ph].

[10] G. C. Nayak, Annals Phys. 325 (2010) 682, arXiv:0905.3931 [hep-ph].

[11] G. C. Nayak, Eur. Phys. J.C59:891,2009.

[12] T. Muta, Foundations of Quantum Chromodynamics, World Scientific lecture notes in physicsVol. 5 .

[13] A. Pilaftsis, Nucl. Phys. B 487 (1997) 467, hep-ph/9607451.

[14] D. M. Capper and G. Leibbrandt, Phys. Rev. D25 (1982) 1002; A. Andrasi and J. C. Taylor, Nucl. Phys. B 192 (1981) 283. 